\title{
A Robust Sandwich Technique with DWT with DB1 and DCT for Image Watermarking with MATLAB
}

\author{
Veeresh Kumar Punjani \\ MTech Scholar \\ Computer Science Engineering Department
}

\author{
Kapil Dev Sharma \\ Assistant Professor \\ Computer Science Engineering Department
}

\begin{abstract}
Over the last few years, growth of internet and technology of media, the protection of copyright have become very vital. To look after multimedia data against illegal copying and transferring, the inclusion of a signal (digital signature, watermark) has become a duty without alter the quality of the original image, the objective of this operation is to identify the holder and care for his intellectual property. Digital watermarking has been projected as a solution to solving the copyright difficulty by introducing indistinguishable data (watermark) into original image. In this paper, we propose a study of digital images watermarking. This study is accomplish by put in watermark in different coefficients of DWT (LH, HL, HH) using det with db1 wavelets family transform by searching the optimal block that have the maximum entropy which can be used to insert the watermark in original image. The experimental results show the different results of PSNR, NCC and IF for each coefficient of DWT and the robustness against most attacks.
\end{abstract}

\section{Keywords}

Watermarking, transformation and wavelets

\section{INTRODUCTION}

Watermarking is a technique used to hide data or identifying information within digital multimedia. Our symposium will spotlight above all on the watermarking of digital images, though digital video, audio, and entry permit are also routinely watermarked. Digital watermarking is attractive popular, particularly for adding undetectable identifying marks, such as author or copyright information. The digital watermarking procedure embeds a signal into the media without appreciably degrading its visual quality. Digital watermarking is a procedure to embed some information called watermark into different kinds of media called Cover Work. Digital watermarking is used to hide the information inside a signal, which cannot be easily extracted by the third party. Its widely used application is copyright protection of digital information. It is diverse from the encryption in the sense that it allows the user to access, view and interpret the signal but protect the possession of the content. Digital watermarking involves embedding a structure in a host signal to "mark" its ownership. Digital watermarks are inside the information so that ownership of the information cannot be claimed by third party. While some watermarks are visible, most watermarks are invisible.

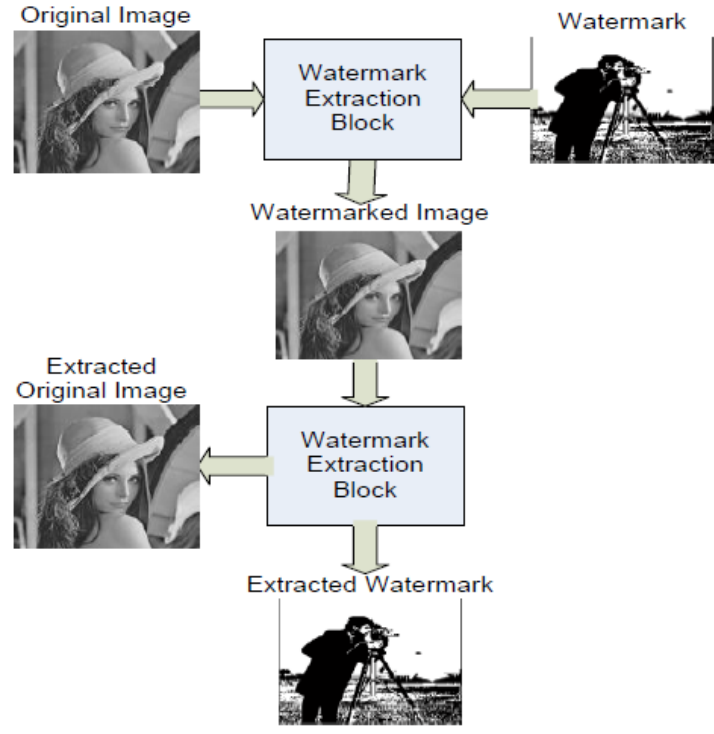

Figure 1: Watermarking Process

\section{APPLICATIONS OF WATERMARING}

Following are the different watermarking applications used in real life areas [18]:

- Copyright protection, it is most likely one of the most commonly used of watermarking application today. Information of copyright owner is embedded in original host image in order to maintain from allege ownership of content.

- $\quad$ Fingerprinting, the fingerprint embeds the appropriate information about permissible recipient in the digital content. This involves embedding distinct watermarks into each of the image and permits the owner to monitor and locate pirated content which is illegally obtained.

- Prevention of unauthorized copying is achieved by embedding the significant information about how frequently an image is likely to be legally copied. An mocking example where the management of a watermark might have avoided the wholesale housebreak of an image is in the Lena image that has been utilized without the permission of original owner.

- Image authentication, in an image authentication application the intention is to detect modifications to the data. The individuality of the image, such as its edges, are embedded and compared with the current images for differences. 


\section{WAVELET APPLICATIONS}

Wavelets can be used in different areas or with different application.

- Detecting Discontinuities and Breakdown Points (I and II)

- Detecting Long-Term Evolution

- Detecting Self-Similarity

- Identifying Pure Frequencies

- $\quad$ Suppressing Signals

- De-Noising Signals

- Compressing Signals

The projected algorithm is executed for each of the below wavelets. wavemngr('read',1)

ans $=$

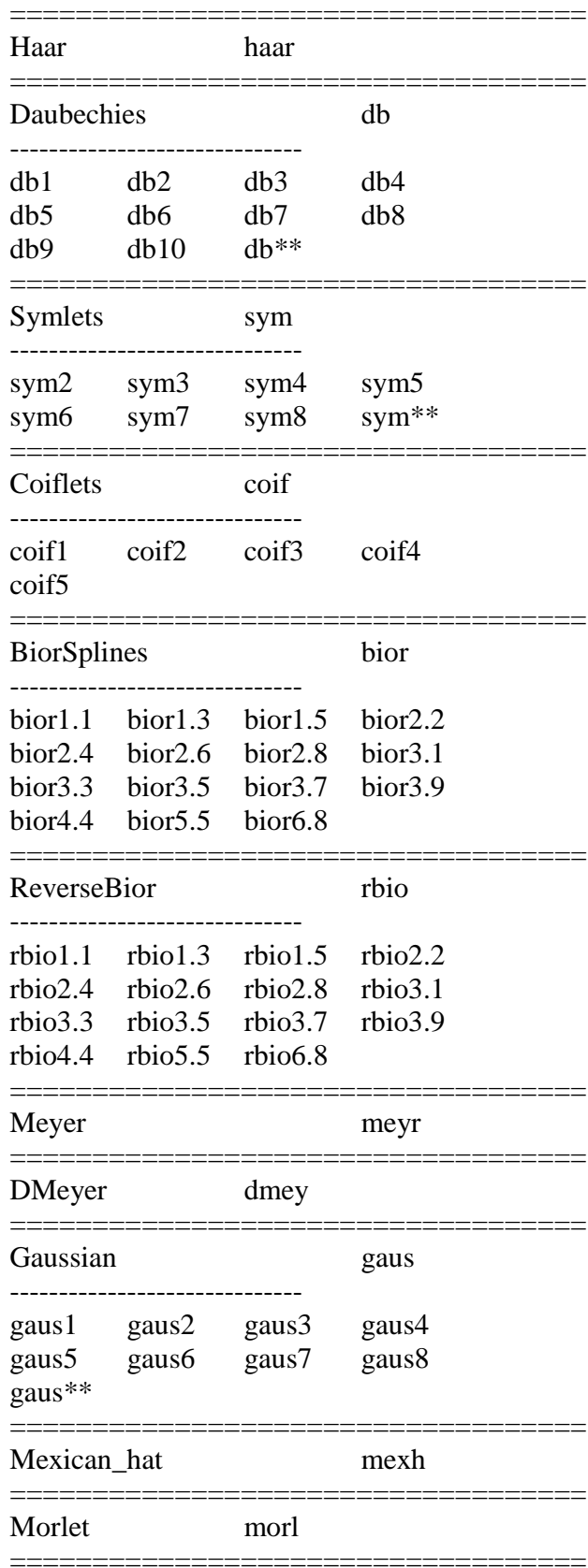

Complex Gaussian

cgau1 cgau2 cgau3 cgau4 cgau5 cgau**

\section{WORKFLOW/WATERMARK ALGORITHM}

The proposed technique had been experimented to measure the performance of system by comparing the result of PSNR, NCC and IF of the system using sandwich template with the hel of transformation in frequency domain.

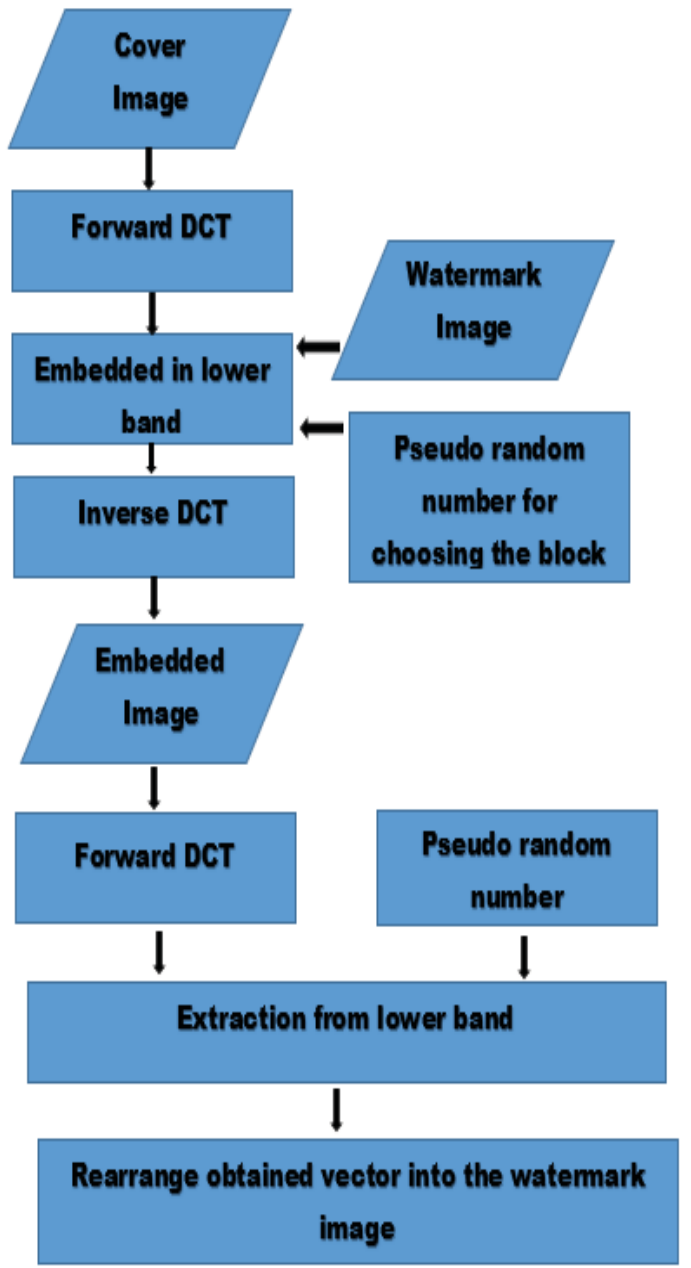

Figure2: Flow chart with dwt 


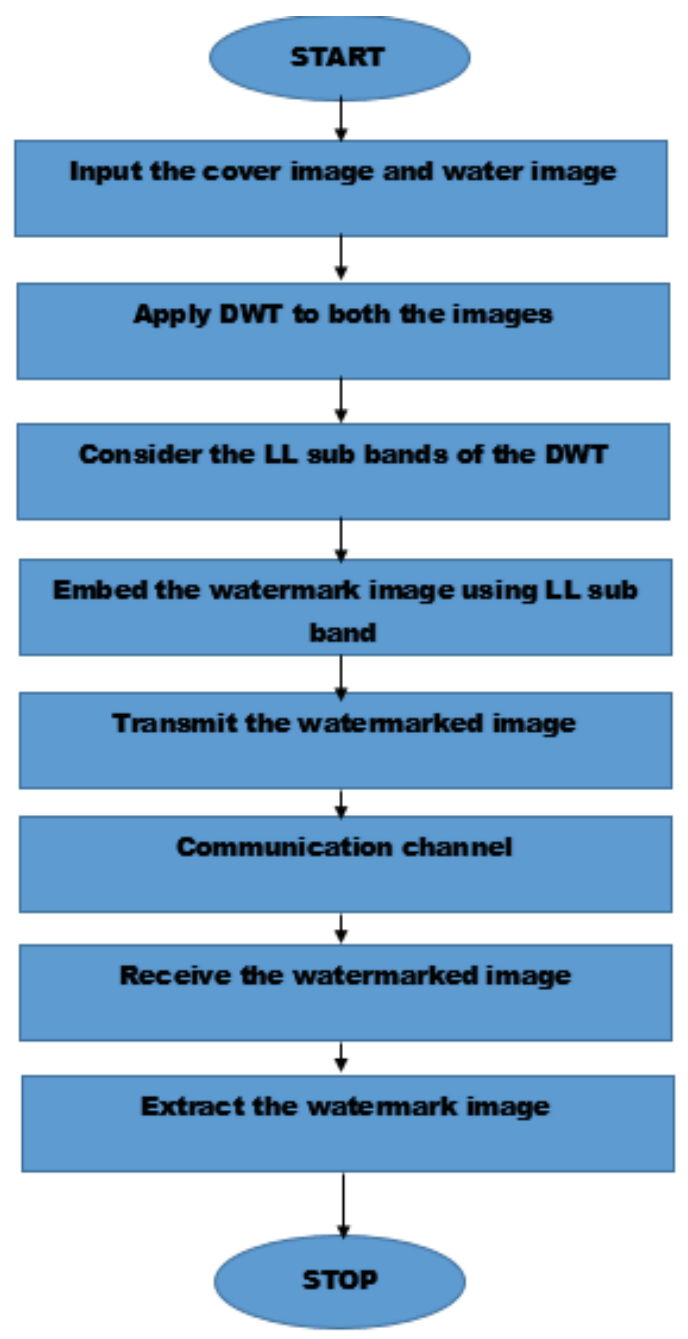

Figure 3: flow chart with det

\section{QUALITY PARAMETER PEAK SIGNAL TO NOISE RATIO (PSNR)}

Notice that, there is no difference between the original and watermarked images in the first and second LSB by using our naked eyes. No distortion occurs for these watermarked images. We found some distortion when we embed the watermark text in the third and fourth LSB and also when we combined them. We got the result after we calculated the Peak signal-to-noise ratio (PSNR).

\subsection{Normalized Cross-Correlation (NCC)}

Normalized cross correlation is the simplest but effective method as a similarity measure, which is invariant to linear brightness and contrast variations. Its easy hardware functioning makes it useful for real-time applications. Or normalized cross correlation is a determine of resemblance of two waveforms as a function of the time lag applied to one of them. The cross correlation is similar in nature to the convolution of two functions.

\section{IMAGE FIDELITY (IF)}

Image fidelity is a measure of the accuracy of the reconstructed sky brightness distribution. A related metric, dynamic range, is a measure of the degree to which imaging artifacts around strong sources are suppressed, which in turn implies a higher fidelity of the on-source reconstruction

\section{Input Test Image:}

Using above proposed algorithms designed a tool in MATLAB and run on MATLAB command, with different test image which is shown in below figure 4 .

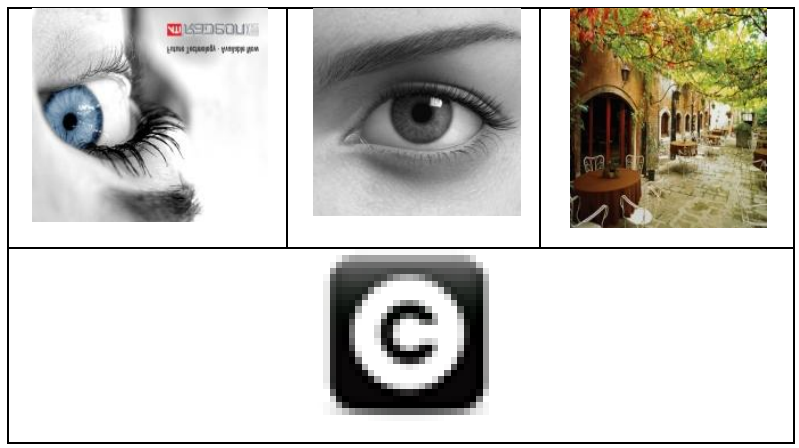

Figure 4: input test Image

\section{RESULT}

In order to evaluate the performance of the proposed method with three input image experiments were performed. In the sets of experiments the performance of the proposed algorithms were recorded with respect to mse, psnr and bit per pixel (bit rate) with same threshold . In below screen shot of matlab command window results (value of PSNR. NCC, IF) are shown.

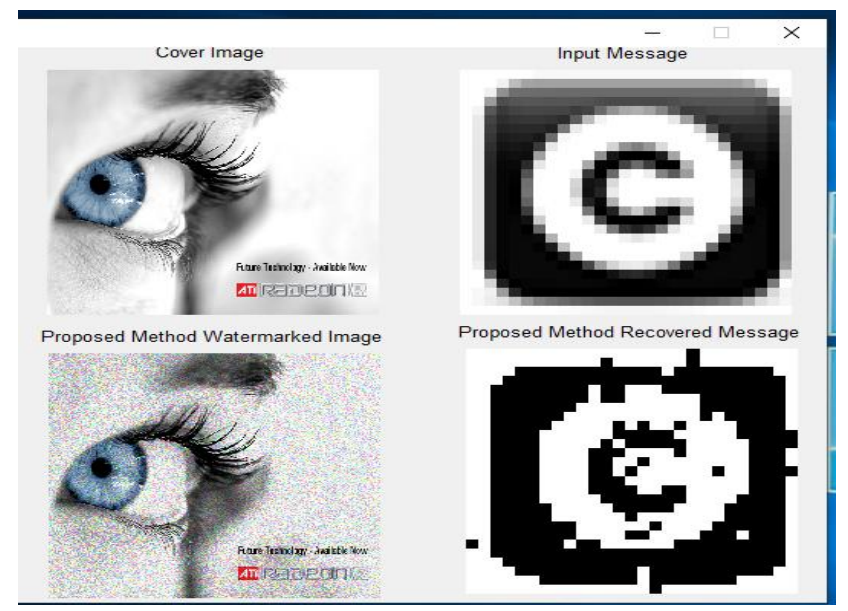

Figure 5: Watermark Image with Test1 image

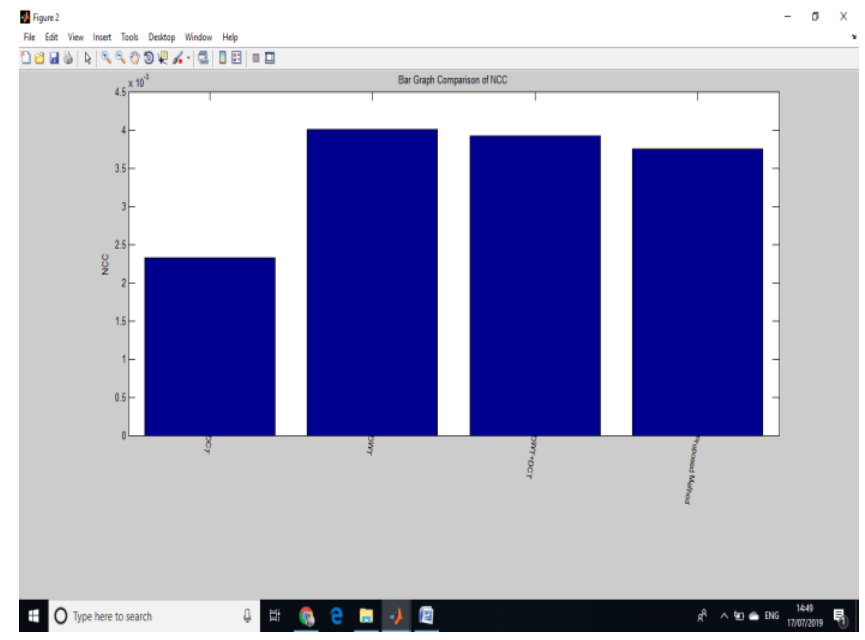

Figure 6: Value of NCC with Test1 image 


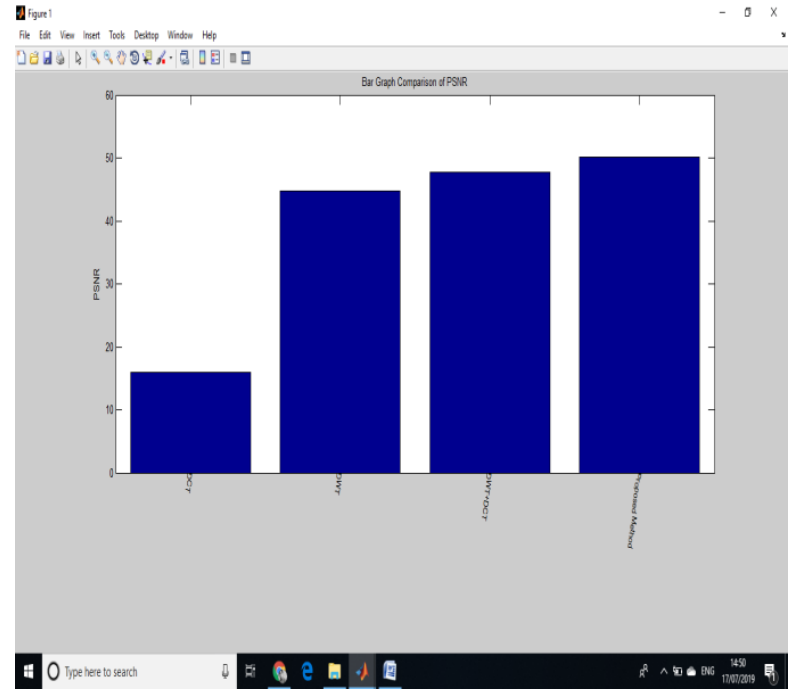

Figure 7: Value of PSNR with Test1 image

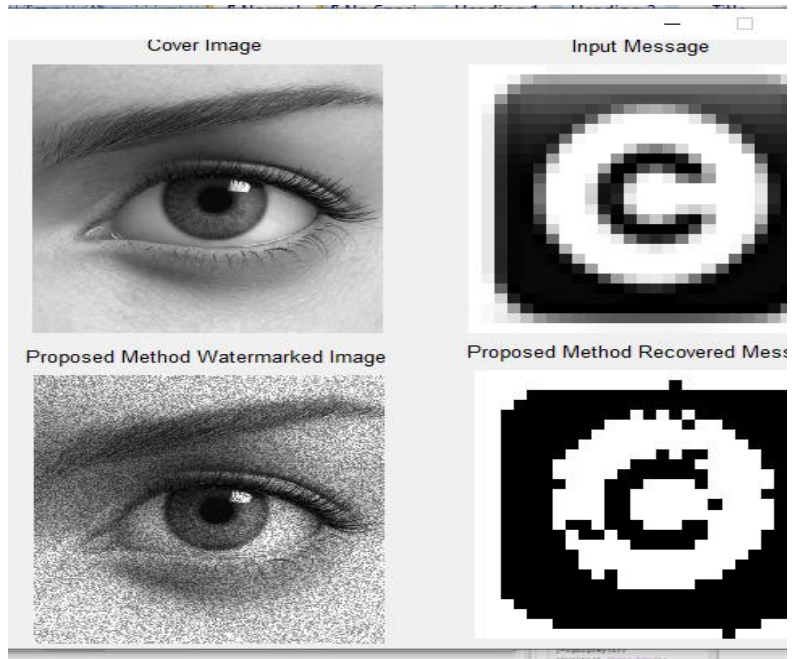

Figure 8: Watermark Image with Test2 image agson

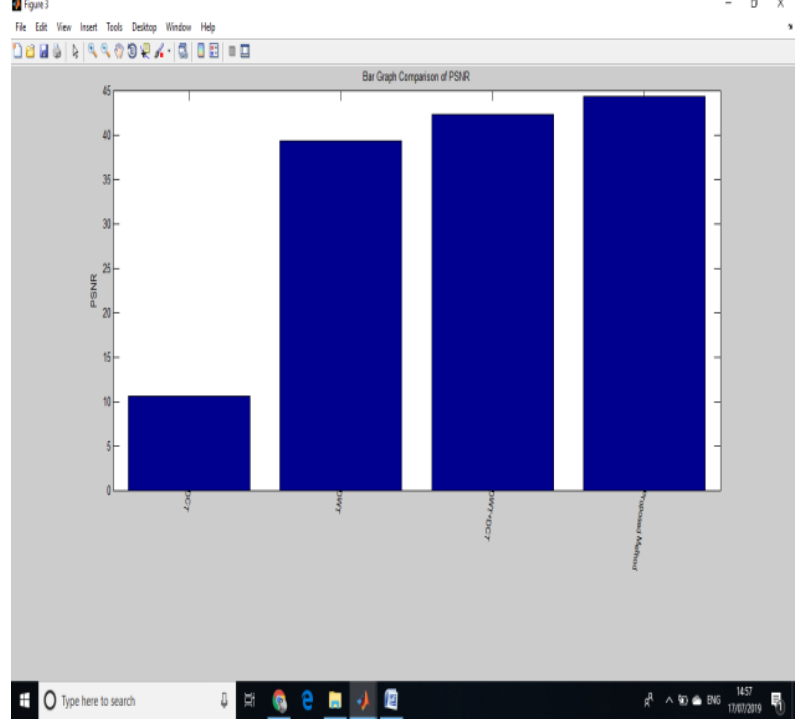

Figure 9: Value of PSNR with Test2 image

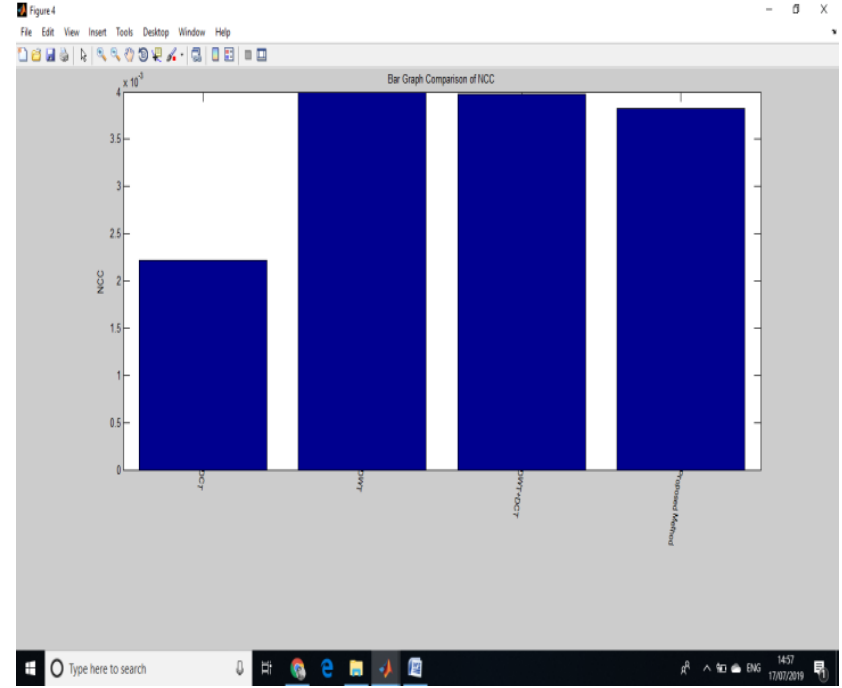

Figure 10: Value of NCC with Test2 image
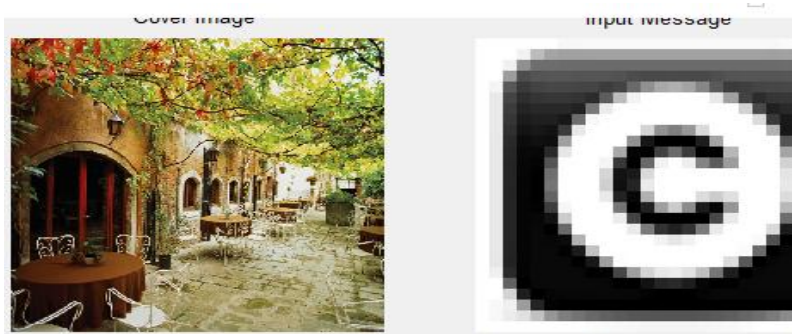

Proposed Method Watermarked Image

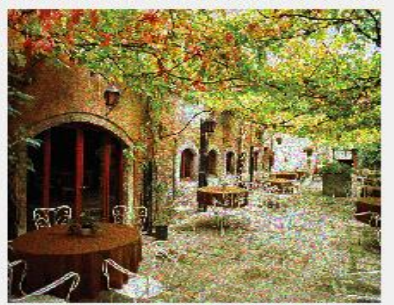

Proposed Method Recovered M€

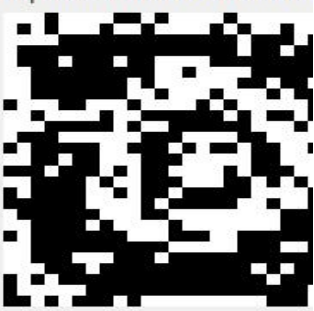

Figure 11: Watermark Image with Test3 image

Ifyout

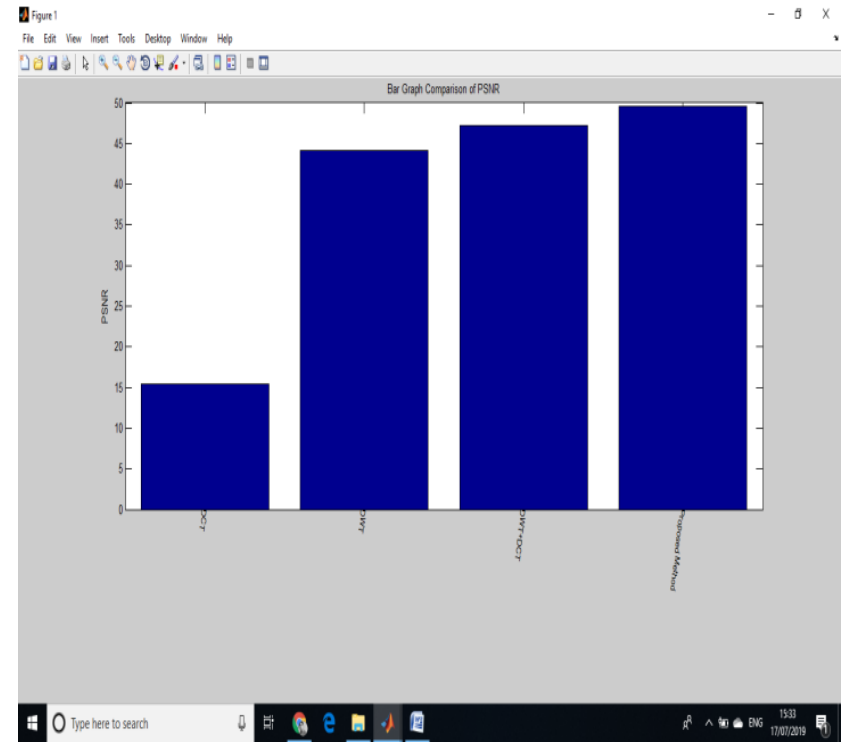

Figure 12: Value of PSNR with Test1 image 


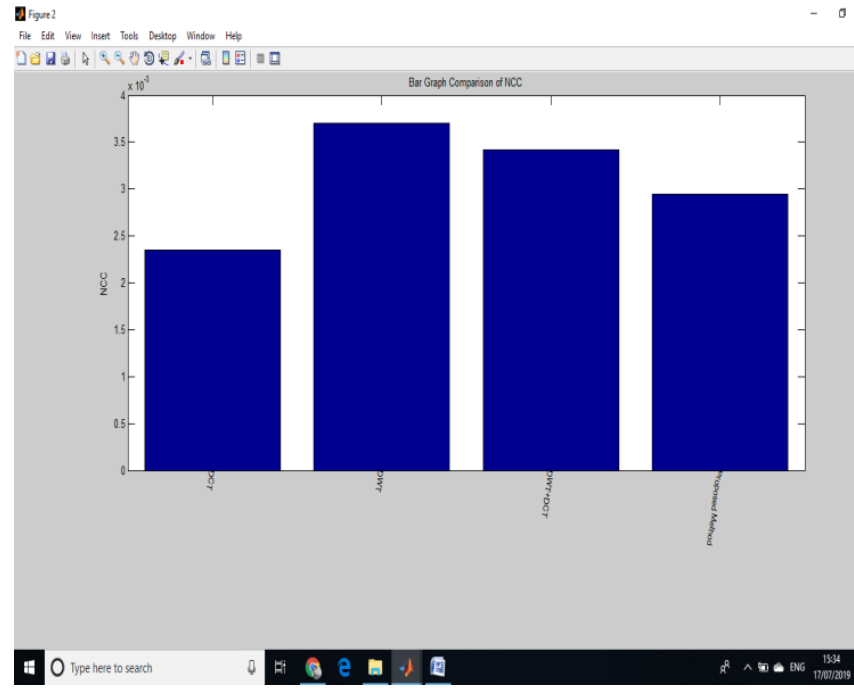

Figure 13: Value of NCC with Test1 image

The proposed algorithm combines the properties of DWT and DCT techniques to increase the robustness and capacity of the algorithm by selecting specifics blocks which have the maximum entropy value. Result in tabular form for the procedure for embedding the watermark is given below.

Table 1: dwt with db1 with Test1 Image

\begin{tabular}{|c|c|c|c|c|}
\hline & Dct & Dwt & Dct+dwt & Proposed Method \\
\hline PSNR & 15.9689 & 44.7370 & 47.6992 & 50.1755 \\
\hline NCC & 0.0023 & 0.0040 & 0.0039 & 0.0038 \\
\hline IF & -0.1133 & $-5.024 \times 10^{-5}$ & $\underset{5}{-2.5401 \times 10^{-}}$ & $-1.4362 \times 10^{-5}$ \\
\hline
\end{tabular}

Table 2: dwt with db1 with Test2 Image

\begin{tabular}{|c|c|c|c|c|}
\hline & Dct & Dwt & Dct+dwt & $\begin{array}{c}\text { Proposed } \\
\text { Method }\end{array}$ \\
\hline PSNR & 10.5647 & 39.2707 & 42.2674 & 44.3327 \\
\hline NCC & 0.0022 & 0.0040 & 0.0040 & 0.0038 \\
\hline IF & -0.2395 & $-3.2257 \times 10^{-4}$ & $-1.6179 \times 10^{-4}$ & $-10056 . \times 10^{-4}$ \\
\hline
\end{tabular}

Table 3: dwt with db1 with Test3 Image

\begin{tabular}{|c|c|c|c|c|}
\hline & Dct & Dwt & Dct+dwt & $\begin{array}{c}\text { Proposed } \\
\text { Method }\end{array}$ \\
\hline PSNR & 15.4586 & 44.1344 & 47.1650 & 49.5784 \\
\hline NCC & 0.0024 & 0.0037 & 0.0034 & 0.0029 \\
\hline IF & -0.4134 & $-1.1925 \times 10^{-5}$ & $-5.9349 \times 10^{-5}$ & $-3.4046 \times 10^{-5}$ \\
\hline
\end{tabular}

\section{CONCLUSION}

Experiments and results were demonstrated in this paper. In this article, we have presented a highly robust watermarking algorithm, in which the DCT is introduced into the hybrid watermarking technique by combining with DWT and DCT. To make a good use of the watermarking technique reduce the influence of watermark embedding, From the study and analysis of Table 1,2 and 3 after applying Test Images of database, came to conclusion that PSNR in proposed method is more accurate or approach to good values approx 50 .
Experimental comparisons suggest that the proposed scheme has little perceptible effect on the original image, and it exhibits better robustness against typical signal processing attacks than other algorithms. In the future work, we will address this issue and apply the proposed method to video.

\section{REFERENCES}

[1] A. M. Eskicioglu and E. J. Delp, "An overview of multimedia content protectionin consumer electronics devices," Signal Processing: Image Communication,vol. 16, no. 7, pp. 681-699, Apr. 2001.

[2] E. T. Lin, A. M. Eskicioglu, R. L. Lagendijk, and E. J. Delp, "Advances in digital video content protection," Proceedings of the IEEE: Special Issue on Advances in Video Coding and Delivery, vol. 93, no. 1, pp. 171-183, Jan. 2005.

[3] Das, C.; Panigrahi, S.; Sharma, V.K.; Mahapatra, K.K. A novel blind robust image watermarking in DCT domain using inter-block coefficient correlation. AEU Int. J. Electron. Commun. 2014, 68, 244-253.

[4] He, Z.F.; Zhang, Y.H. Fusion on the wavelet coefficients scale-related for double encryption holographic halftone watermark hidden technology. IEICE Trans. Inf. Syst. 2015, E98-D, 1391-1395.

[5] Hu, Y.P.; Wang, Z.J.; Liu, H.; Guo, G.J. A geometric distortion resilient image watermark algorithm based onDWT-DFT. J. Softw. 2011, 6, 1805-1812. .

[6] Liu, R.Z.; Tan, T.N. An SVD-based watermarking scheme for protecting rightful ownership. IEEE Trans. Multimed. 2002, 4, 121-128.

[7] Lai, C.C.; Tsai, C.C. Digital image watermarking using discrete wavelet transform and singular value decomposition. IEEE Trans. Instrum. Meas. 2010, 59, 3060-3063.

[8] Gupta, A.K.; Raval, M.S. A robust and secure watermarking scheme based on singular values replacement. Sadhana 2012, 37, 425-440. .

[9] Singh, A.K.; Kumar, B.; Dave, M.; Mohan, A. Robust and imperceptible dual watermarking for telemedicine applications. Wirel. Pers. Commun. 2015, 80, 14151433.

[10] Singh, A.K. Improved hybrid algorithm for robust and imperceptible multiple watermarking using digital images. Multimed. Tools Appl. 2017, 76, 8881-8900.

[11] Singh, A.K.; Dave, M.; Mohan, A. Hybrid technique for robust and imperceptible image watermarking in DWTDCT-SVD domain. Natl. Acad. Sci. Lett. 2014, 37, 351-358.

[12] Singh, A.; Tayal, A. Choice of wavelet from wavelet families for DWT-DCT-SVD image watermarking. Int. J.Comput. Appl. 2012, 48, 9-14. .

[13] Roy, S.; Pal, A.K. An SVD based location specific robust color image watermarking scheme using RDWT and Arnold Scrambling. Wirel. Pers. Commun. 2018, 98, 2223-2250.

[14] Laur, L.; Rasti, P.; Agoyi, M.; Anbarjafari, G. A robust color image watermarking scheme using entropy and QR decomposition. Radioengineering 2015, 24, 1025 1032 . 
[15] Mishra, A.; Agarwal, C.; Sharma, A.; Bedi, P. Optimized gray-scale image watermarking using DWTSVD and firefly algorithm. Expert Syst. Appl. 2014, 41, 7858-7867.

[16] Yang, X.S. Multiobjective firefly algorithm for continuous optimization. Eng. Comput. 2013, 29, 175184.

[17] Md. Maklachur Rahman, "A DWT, DCT AND SVD BASED WATERMARKING TECHNIQUE TO PROTECT THE I MAGE PIRACY" International Journal of Managing Public Sector Information and Communication Technologies (IJMPICT) Vol. 4,No. 2, June 2013.

[18] Vijaya K. Ahiret, Vivek Kshirsagar, "Robust Watermarking Scheme Based on Discrete Wavelet
Transform (DWT) and Discrete Cosine Transform (DCT) for Copyright Protection of Digital Images", IJCSNS International Journal of Computer Science and NetworkSecurity, VOL. I I No.8, August 20 I I.

[19] Mei Jiansheng, Li Sukang and Tan Xiaomei, "A Digital Watermarking Algorithm Based On DCT and DWT", Proceedings of the 2009 International Symposium on Web Information Systems and Applications (WISA'09) Nanchang,P.R. China, May 22-24,2009, pp. 104-107.

[20] Nikita Kashyap, G. R. SINHA, "Image Watermarking Using 3-Level Discrete Wavelet Transform (DWT)", U.Modern Education and Computer Science, 2012, 3, 50-56 Published Online April 2012 in MECS, DOT: 10.5815/ijmecs.2012.03.07 